\title{
EFEITO DE EXTRATOS AQUOSOS DE ESPÉCIES VEGETAIS SOBRE A MULTIPLICAÇÃO DE ROTYLENCHULUS RENIFORMIS LINFORD \& OLIVEIRA
}

\section{C.G. Gardiano ${ }^{2}$, S.P. Muramoto ${ }^{1 \dagger}$, A.A. Krzyzanowiski ${ }^{1}$, W.P. de Almeida ${ }^{1}$, O.J.G.A. Saab ${ }^{2}$}

${ }^{1}$ Instituto Agronômico do Paraná, CP 481, CEP 86001-970, Londrina, PR, Brasil. E-mail: cris_gardiano@ yahoo.com.br

\section{RESUMO}

\begin{abstract}
A busca por medidas alternativas no controle de fitonematoides vem sendo estimulada, e a aplicação de extratos vegetais pode tornar-se uma medida viável para pequenas aéreas. Avaliouse o efeito da adição ao solo de extratos aquosos de oito espécies vegetais sobre a multiplicação de Rotylenchulus reniformis. O experimento foi conduzido em condições de casa de vegetação com temperaturas máxima e mínima de $34,2^{\circ} \mathrm{C}$ e $25,6^{\circ} \mathrm{C}$, respectivamente. Plantas de algodoeiro foram inoculadas com 4.000 ovos do nematoide e, em seguida, adicionou-se $20 \mathrm{~mL}$ dos extratos aquosos obtidos de folhas de Crotalaria spectabilis, Arctium lappa, Plectranthus barbatus, Rosmarinus officinalis, Origanum vulgare, Cajanus cajan, Mucuna aterrima e Momordica charantia. O inoculo foi obtido de populações puras, multiplicadas em plantas de algodoeiro, mantidas em casa de vegetação. Após 60 dias, avaliou-se o número de ovos, altura das plantas, diâmetro do caule e peso fresco da parte aérea e das raízes. Os extratos de $C$. cajan, O. vulgare, $M$. aterrima e $M$. charantia reduziram o número de ovos em 28, 28, 44 e 60\%, respectivamente. Para o diâmetro do caule os extratos de M. aterrima e C. cajan diferiram da testemunha e demais tratamentos. Com relação ao peso da parte aérea, os extratos de C. cajan, P. barbatus, M. aterrima e C. spectabilis diferiram da testemunha e demais tratamentos. Portanto, os extratos de Mucuna aterrima, C. cajan, O. vulgare e M. charantia podem ser uma opção para o controle de $R$. reniformis.
\end{abstract}

PALAVRAS-CHAVE: Nematoide reniforme, controle alternativo, compostos naturais, plantas medicinais.

\begin{abstract}
THE EFFECT OF AQUEOUS EXTRACTS OF PLANT SPECIES ON THE MULTIPLICATION OF ROTYLENCHULUS RENIFORMIS LINFORD \& OLIVEIRA. The search for alternative measures for the control of nematodes has been stimulated, and the application of plant extracts can become a viable measure for small farmers. This study evaluated the effect on the multiplication of Rotylenchulus reniformis when aqueous extracts of eight plant species were added to the soil. The experiment was conducted under greenhouse conditions with maximum and minimum temperatures of 34.2 and $25.6^{\circ} \mathrm{C}$, respectively. Cotton plants were inoculated with 4,000 nematode eggs and then a volume of $20 \mathrm{~mL}$ of aqueous extracts obtained from leaves of Crotalaria spectabilis, Arctium lappa, Plectranthus barbatus, Rosmarinus officinalis, Origanum vulgare, Cajanus cajan, Mucuna aterrima and Momordica charantia was added. The inoculum was obtained from pure populations multiplied in cotton plants, kept in a greenhouse. After 60 days the number of eggs, plant height, stem diameter and fresh weight of shoots and roots was evaluated. The extracts of Cajanus cajan, Origanum vulgare, Mucuna aterrima, and Momordica charantia reduced the number of eggs by $28,28,44$ and $60 \%$ respectively. In relation to stem diameter, the extracts of $M$. aterrima and C. cajan differed from the control and the other treatments. In relation to the weight of the shoot, extracts of C. cajan, P. barbatus, M. aterrima and C. spectabilis differed from the control and the other treatments. Therefore, the extracts of M. aterrima, C. cajan, O. vulgare and M. charantia can serve as options for the control of $R$. reniformis.
\end{abstract}

KEY WORDS: Reniform nematode, alternative control, natural compounds, medicinal plants.

${ }^{2}$ Universidade Estadual de Londrina, Centro de Ciências Agrárias, Departamento de Agronomia, Londrina, PR, Brasil. †In memorian. 


\section{INTRODUÇÃO}

No controle de nematoides, o produto químico muitas vezes é a única opção para o produtor que exige uma resposta rápida para o seu problema, levando-o frequentemente ao uso indiscriminado destes produtos, com consequências desastrosas. Por isso, a busca por novas alternativas de controle vem sendo estimulada, e a utilização de extratos vegetais pode ser uma dessas. Já que o uso destes extratos com propriedades nematicidas no controle de fitonematoides representa mais uma alternativa para os pequenos produtores, com valor prático eeconômico, e sem riscos de contaminação do ambiente, onde várias pesquisas têm demonstrado o efeito nematicida dos extratos de diferentes plantas, quando aplicados diretamente ao solo (CHITwOOD, 2002).

Extratos botânicos apresentam vantagens sobre pesticidas sintéticos como: são menos concentrados e menos tóxicos, são biodegradados rapidamente, possuem um amplo modo de ação e são derivados de recursos renováveis, segundo QuARLES (1992).

O estudo com extratos vegetais sobre fitonematoides vem sendo feito por diversos autores e apresentado grande potencial como matéria-prima para produção de nematicidas naturais (LEWIS; PAPAVIZAS, 1971; LazZeri et al., 1993; FerRIZ; ZeNGH, 1999). No entanto, estudo do efeito de extratos de plantas sobre o nematoide Rotylenchulus reniformis são poucos ou quase inexistentes em literatura. O objetivo desse trabalho foi de avaliar o efeito da adição no solo dos extratos aquosos de oito espécies vegetais sobre a população de $R$. reniformis.

\section{MATERIAL E MÉTODOS}

O experimento foi conduzido em condições de casa de vegetação do Instituto Agronômico do Paraná, Londrina, PR, no período de 20/11/2009 a 20/1/2010, com médias de temperaturas máxima e mínima de $34,2^{\circ} \mathrm{C}$ e $25,6^{\circ} \mathrm{C}$.

As espécies de plantas utilizadas foram: alecrim (Rosmarinus officinalis L.), bardana (Arctium lappa L.), boldo (Plectranthus barbatus Andrews), Crotalaria spectabilis Roth, Guandu [Cajanus cajan (L.) Millsp.], melão de São Caetano (Momordica charantia L.), mucuna preta (Mucunaaterrima Piper \& Tracer), orégano (Origanum vulgare L.).

Os extratos aquosos das folhas foram obtidos seguindo o método descrito por FERRIS; ZHENG (1999), no qual se misturou $1 \mathrm{~g}$ de folhas secas de cada espécie de planta com $10 \mathrm{~mL}$ deágua destilada, mantendoem repouso por 24 horas. Posteriormente, essa mistura foi filtrada em gaze e colocada em erlenmeyers cobertos com papel alumínio, identificados e utilizados logo em seguida.
Mudas de algodoeiro (Gossipyum hirsutum) da cultivar CD 401, com 20 dias de idade, foram transplantadas para vasos de plástico com capacidade de $0,5 \mathrm{~kg}$, contendo uma mistura de solo e areia na proporção de 2:1 (v:v), previamente esterilizado. Em seguida, o solo foi infestado com uma suspensão de 4.000 ovos de $R$. reniformis. O inóculo foi extraído utilizando a metodologia de BONETI; FERRAZ (1981). No mesmo dia em que o solo foi infestado, $20 \mathrm{~mL}$ dos extratos vegetais foram adicionados ao solo de cada vaso, na forma de rega. Na testemunha foram aplicados $20 \mathrm{~mL}$ de água destilada ao solo. A aplicação foi realizada quinzenalmente por um período de 60 dias.

Aos 60 dias após a inoculação, foram avaliados o número de ovos por sistema radicular, o peso fresco do sistema radicular e da parte aérea, a altura e o diâmetro de cada planta.

O delineamento estatístico utilizado foi o inteiramente casualizado, com 9 tratamentos e 8 repetições. Os dados obtidos foram submetidos à análise de variância e, quando necessário, ao teste de comparação de médias de Scott-Knot, ao nível de 5\% de probabilidade estatística. Os resultados obtidos da avaliação do número de ovos por sistema radicular foram transformados para $\log _{10}(x)$ e os valores obtidos para peso fresco da raiz para " $(x+k)^{\wedge} 1 / 2$ " com $\mathrm{k}=0,1$.

\section{RESULTADOS E DISCUSSÃO}

A adição ao solo dos extratos das plantas testadas nesse experimento não apresentou nenhum efeito sobre o peso fresco do sistema radicular e nem na altura das plantas (Tabela 1), no entanto, os extratos de guandu e mucuna preta promoveram um aumento no diâmetro do caule, diferindo da testemunha (Tabela 1). Os extratos de C. spectabilis, boldo, guandu e mucuna preta promoveram um aumento no peso fresco da parte aérea das plantas diferindo da testemunha (Tabela 1).

Com relação ao número de ovos, a adição ao solo dos extratos de guandu, orégano, mucuna preta em melãodeSão Caetano promoveram redução(Tabela1). No entanto, o extrato de Melão de São Caetano foi o que apresentou melhor efeito sobre a população, com uma redução de $60 \%$ (Tabela 1).

Diversas espécies de plantas vêm sendo utilizadas sobre a forma de incorporação de suas partes vegetais ou sob a forma de extratos, sendo ambas as formas aplicadas ao solo sobre diversas espécies de nematóides (HOAN; DAVIDE, 1979; CANNAYANE; RAJENDRAN, 2003; LOPES, 2004).

Quanto ao guandu, vários trabalhosjá foram feitos demonstrandoque possui propriedades antagonistas em relação diferentes espécies de nematoides, como 
Pratylenchus penetrans (HAROON; ABADIR, 1989); M. javanica (Asmus; Ferraz, 1988; Costa; Ferraz, 1990), M incognita (HAROON; AbADIR, 1989), mas todos na forma de rotação ou testando sua resistência em relação a estes nematóides; na forma de extratos no controle de $R$. renifomis não foram encontrados nenhum relato.

O orégano foi outra espécie que demonstrou bons resultados nesse trabalho (Tabela 1), contudo, estudos relacionando o uso desta planta no controle de nematoides não foram encontrados na literatura, no entanto, seus constituintes químicos principais são taninos, timol, fenóis, origaneno e caneacol; e como se trata de uma planta aromática, seu óleo essencial possui propriedades variadas, entre elas, vermífuga (MARTINs et al., 2003).

LOPEs et al. (2005), avaliaram o efeito da adição ao solo e pulverização foliar de extratos de sementes e de folhas de mucuna preta, sobre Meloidogyne incognita e M. javanica. Verificaram que a adição ao solo dos extratos de sementes apresentou uma redução de $47 \%$ na reprodução apenas de $M$. javanica em relação a testemunha; e a aplicação via pulverização foliar dos extratos de sementes e de folhas de mucuna preta mostrou uma redução de $29,7 \%$ e $26,5 \%$, respectivamente, no número de galhas apenas para $M$. incognita.

O Melão de São Caetano foi a espécies que demonstrou melhor eficiência em relação ao controle de $R$. reniformis nesse trabalho (Tabela 1), é uma planta que apresenta como constituinte químico em suas folhas momordicina (alcaloide), momordicripina e ácido momórdico (MARTINs et al., 2003). No entanto, nenhuma referencia relacionando o uso de extratos de suas folhas no controle de $R$. reniformis foi encontrado na literatura, porém, essa planta já foi avaliada para $M$. incognita, em que a infusão desta apresentou efeito nematicida sobre esse nematoide com uma porcentagem de juvenis mortos acima de 80\% (DiAs et al., 2000).

Estudos relacionando o controle de $R$. reniformis ao uso de extratos vegetais não foram encontrados na literatura, por isso a dificuldade de se encontrar trabalhos para serem confrontados com os resultados obtidos nesse experimento.

Diante desses resultados, é possível concluir que extratos aquosos de guandu, orégano, mucuna preta e melão de São Caetano podem ser utilizados como forma alternativa de controle de $R$. reniformis. Sendo assim, estudos posteriores são necessários para se conhecer os princípios ativos dessas plantas responsáveis pela ação nematicida, e sua eficiência em condição de campo; e para verificar se existe especificidade em relação ao nematoide estudado.

\section{REFERÊNCIAS}

ASMUS, R.M.F.; FERRAZ, S. Antagonismo de algumas espécies vegetais, principalmente leguminosas, à Meloidogyne javanica. Fitopatologia Brasileira, v.13, n.1, p.20-24, 1988.

BONETI, J.I.S.; FERRAZ, S. Modificação do método para extração de ovos para Meloidogyne exigua em raízes de cafeeiro. Fitopatologia Brasileira, v.6, p.553, 1981.

CANNAYANE, I.; RAJENDRAN, G. Penetration of Meloidogyne incognita (race 3 ) in tomato and brinjal roots

Tabela 1 - Número de ovos por sistema radicular, peso fresco da raiz e da parte aérea, altura e diâmetro do caule de plantas de algodoeiro tratadas quinzenalmente com extratos aquosos de 8 espécies vegetais, adicionados ao solo, aos 60 dias após a infestação do solo com 4.000 ovos de Rotylenchulus reniformis.

\begin{tabular}{|c|c|c|c|c|c|c|}
\hline \multirow{2}{*}{ Tratamentos } & \multirow{2}{*}{$\begin{array}{c}\begin{array}{c}\text { Altura das } \\
\text { plantas }\end{array} \\
(\mathrm{cm})\end{array}$} & \multirow{2}{*}{$\begin{array}{c}\begin{array}{c}\text { Diâmetro } \\
\text { do caule }\end{array} \\
(\mathrm{cm})\end{array}$} & \multicolumn{2}{|c|}{ Peso fresco $(\mathrm{g})$} & \multirow{2}{*}{ № de ovos ${ }^{2}$} & \multirow{2}{*}{$\begin{array}{l}\text { Porcentagem } \\
\text { de redução }\end{array}$} \\
\hline & & & Sistema radicular ${ }^{1}$ & Parte aérea & & \\
\hline Crotalaria spectabilis & $26,50 \mathrm{a}$ & $0,53 \mathrm{~b}$ & $5,11 \mathrm{a}$ & $9,46 \mathrm{a}$ & $7.137,50 \mathrm{a}$ & $+37 \%$ \\
\hline Bardana & $28,44 \mathrm{a}$ & $0,51 \mathrm{~b}$ & $3,62 \mathrm{a}$ & $7,88 \mathrm{~b}$ & $6.258,75 \mathrm{a}$ & $+21 \%$ \\
\hline Boldo & 27,88 a & $0,54 \mathrm{~b}$ & $5,25 \mathrm{a}$ & $10,35 \mathrm{a}$ & $5.600,00 \mathrm{a}$ & $+8 \%$ \\
\hline Alecrim & $26,69 \mathrm{a}$ & $0,48 \mathrm{~b}$ & $3,45 \mathrm{a}$ & $7,03 \mathrm{~b}$ & $4.975,00 \mathrm{a}$ & $-4 \%$ \\
\hline Guandu & $28,94 \mathrm{a}$ & $0,60 \mathrm{a}$ & $4,29 \mathrm{a}$ & 11,79 a & $3.731,25 \mathrm{~b}$ & $-28 \%$ \\
\hline Orégano & 24,81 a & $0,53 \mathrm{~b}$ & $3,98 \mathrm{a}$ & $8,61 \mathrm{~b}$ & $3.737,50 \mathrm{~b}$ & $-28 \%$ \\
\hline Mucuna Preta & $27,13 \mathrm{a}$ & $0,60 \mathrm{a}$ & $5,11 \mathrm{a}$ & $9,92 \mathrm{a}$ & $2.887,50 \mathrm{~b}$ & $-44 \%$ \\
\hline Melão de São Caetano & 27,19 a & $0,54 \mathrm{~b}$ & $4,75 \mathrm{a}$ & $7,82 \mathrm{~b}$ & $2.100,00 \mathrm{c}$ & $-60 \%$ \\
\hline Testemunha & $27,31 \mathrm{a}$ & $0,48 \mathrm{~b}$ & $5,76 \mathrm{a}$ & $8,20 \mathrm{~b}$ & $5.187,50 \mathrm{a}$ & $0 \%$ \\
\hline CV $(\%)$ & 7,55 & 10,92 & 19,39 & 20,41 & 4,9 & \\
\hline
\end{tabular}

Média de 8 repetições.

${ }^{1}$ Valores transformados para “ $(\mathrm{x}+\mathrm{k})^{\wedge} 1 / 2$ " $\operatorname{com} \mathrm{k}=0,1$.

${ }^{2}$ Valores transformados para $\log x$ na base 10.

${ }^{3}(+)$ indica aumento na população e (-) indica redução da população do nematoide. 
trated with botanical extracts. Indian Journal of Plant Protection, v.31, n.2, p.84-86. 2003.

CHITWOOD, D.J. Fitoquímico estratégias com base para o controle de nematóides. Annual Review of Phytopathology, v.40, p.221-249, 2002.

COSTA, D.C.; FERRAZ, S. Avaliação do efeito antagônico de algumas plantas, principalmente de inverno, à Meloidogyne javanica. Nematologia Brasileira, v.14, p.61-70, 1990.

DIAS,C.R.; SCHWAN, A.V.; EZEQUIEL, D.P.; SARMENTO, M.C.; FERRAZ, S. Efeito de extratos aquosos de plantas medicinais na sobrevivência de juvenis de Meloidogyne incognita. Nematologia Brasileira, v.24, n.2, p.203-210, 2000.

FERRIS, H.; ZHENG, L. Plant sources of chinese herbal remedies: Effects on Pratylenchus vulnus and Meloidogyne javanica. Journal of Nematology, v.31, p.241-263, 1999.

HAROON, S.A.; ABADIR, S.H. The effect of four summer legume cover crops on the population level of Meloidogyne incognita, Pratylenchus penetrans and Trichodorus christie. Journal of Agricultural Sciences, v.20, n.2, p.25-35, 1989.

HOAN, L.T.; DAVIDE, R.G. Nematicidal properties of root extracts of seventeen plant species on Meloidogyne incognita. Philipine Agriculturist, v. 62, p.285-295, 1979.
LAZZERI, L.; TACCONI, R.; PALMIERI, S. In vitro activity of some glucosinolates and their reaction products toward a population of the nematode Heterodera schachtii. Journal of Agriculture and Food Chemistry, v.41, p.825-829, 1993.

LEWIS, J.A.; PAPAVIZAS, G.C. Effect of sulfur-containing volatile compounds and vapors from cabbage decomposition on Aphanomyces euteiches. Phytopathology, v.61, p.208-214, 1971.

LOPES, E.A.; FERRAZ, S.; FREITAS, L.G.; FERREIRA, P.A.; AMORA, D.X. Efeito de extratos aquosos de mucuna preta e de manjericão sobre Meloidogyne incognita e $M$. javanica. Nematologia Brasileira, v.29, n.1, p.67-74, 2005.

LOPES, E.A. Potencial de extratos aquosos e da incorporação ao solo de Mucuna Preta (Mucuna pruriens var. utilis) para o controle do nematóide das galhas. 2004. 54f. Dissertação (Mestrado) - Universidade Federal de Viçosa, Viçosa, MG, 2004.

MARTINS, E.R.; CASTRO, D.M. de; CASTELLANI, D.C.; DIAS, J.E. Plantas medicinais. Viçosa, MG: Editora UFV, 2003.

QUARLES, W. Botanical pesticides from Chenopodium. IPM Practitioner, v.14, n.2, p.1-11, 1992.

Recebido em 5/10/10

Aceito em 30/10/11 